\title{
Uniaxial and biaxial ratchetting in piping materials-experiments and analysis
}

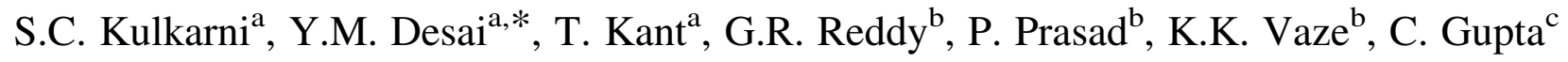 \\ ${ }^{a}$ Department of Civil Engineering, Indian Institute of Technology Bombay, Powai, Mumbai 400 076, India \\ ${ }^{\mathrm{b}}$ Reactor Safety Division, Bhabha Atomic Research Centre, Trombay, Mumbai 400 085, India \\ ${ }^{\mathrm{c}}$ Material Science Division, Bhabha Atomic Research Centre, Trombay, Mumbai 400 085, India
}

\begin{abstract}
The performance of the Chaboche kinematic hardening model has been evaluated in this paper to predict the ratchetting responses for a broad set of uniaxial and biaxial loading histories. The investigations have been performed with reference to both uniaxial and biaxial experimental data, viz. (a) strain and stress controlled uniaxial tests on tensile specimens; (b) biaxial tests on straight pipes with constant internal pressure and cyclic bending load; and (c) a shake table test on elbow. The parameters of the Chaboche model have been calculated from the uniaxial strain controlled stable hysteresis loop. Amongst the various parameters in the Chaboche model, it has been found that the selection of the value of $\gamma_{3}$ plays a crucial role in achieving better simulation. The Chaboche model was observed to predict complete shakedown for $\gamma_{3}=0$. On the other hand, the model closely simulated the experimental results for $\gamma_{3}=9$. The same parameters have been used to analyze the biaxial loading condition. Ratchetting simulation studies by the Chaboche model have resulted in reasonably good agreement with experiments.
\end{abstract}

Keywords: Uniaxial and biaxial ratchetting; Chaboche model; Uniaxial and biaxial experiments

\section{Introduction}

Piping networks are often employed in various industrial applications, including nuclear power plants. Generally, piping systems in a nuclear power plant are designed for normal operation loads (pressure) along with cyclic loads, such as earthquake. This cyclic loading on the piping with nonzero mean static stress results in either structural shakedown or ratchetting. With the occurrence of structural shakedown, the dissipated energy in the whole structure remains bounded after initial plastic flow and the structure responds in a purely elastic manner to the applied variable loads. On the other hand, the ratchetting phenomenon is defined as a cycle-by-cycle accumulation of plastic strain with the application of cyclic load characterized by constant stress amplitude with a nonzero mean stress. After a sufficient number of cycles, the total strain (and therefore displacement) becomes so large that the original shape of the structure is altered, thereby making the structure unserviceable. Typical ratchetting and shakedown responses under repetitive loading are shown in Fig. 1.

The ratchetting response of a material is significantly influenced by the stress history, which in turn depends on the external loads as well as on the geometry of a pipe. Also, ratchetting depends on the anisotropic property of the material due to different strain-hardening curves in tension and compression. This differential strain hardening causes structures to ratchet under cyclic loading. The most well known nonlinear kinematic hardening model has been proposed by Armstrong-Frederick [1]. This model includes a kinematic hardening rule containing a 'recall term' which incorporates the fading memory effects of the strain path and essentially makes the rule nonlinear in nature. Also, the anisotropy property of the tension and compression curves has been considered in this model, which produces a change in shape between the forward and the reverse loading paths. Therefore, the stress-strain hysteresis loop does not close and the ratchetting phenomenon occurs. However, the stressstrain loop produced by this model deviates significantly 


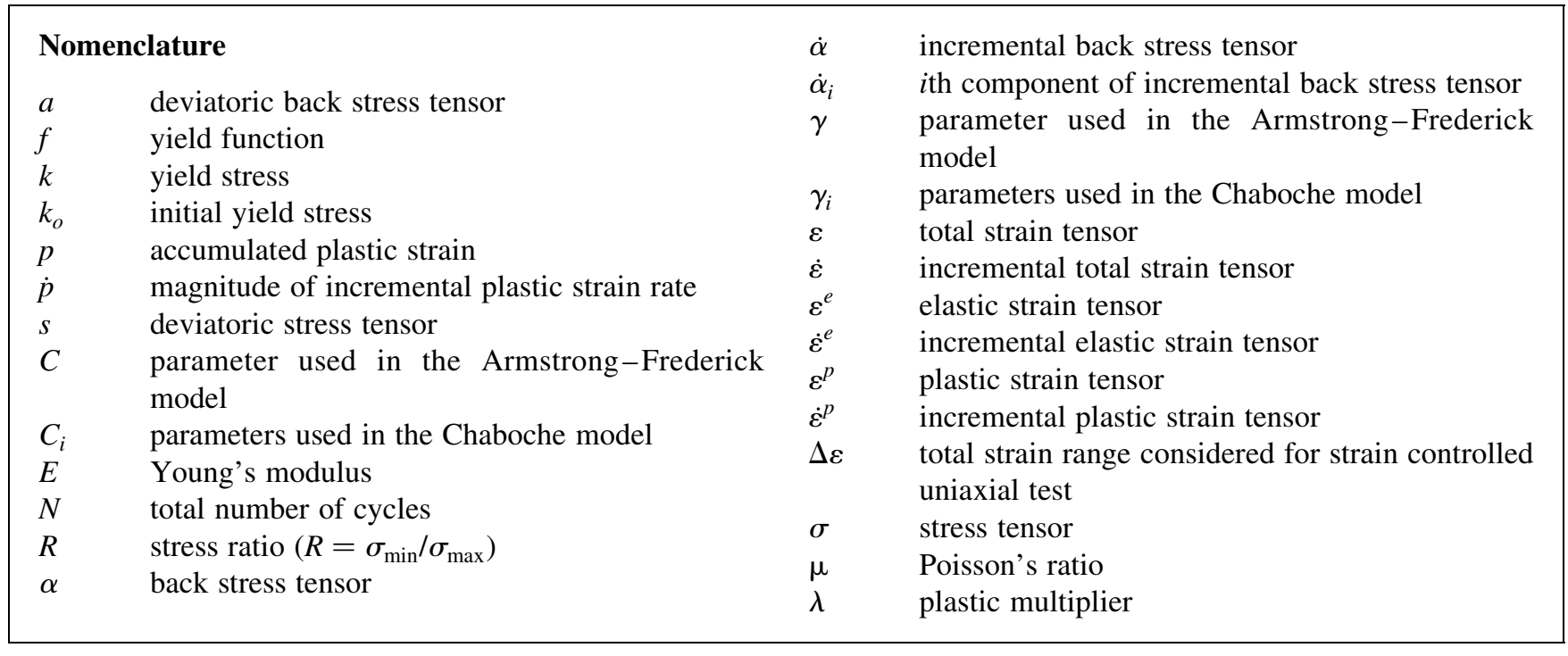

from experiment and the ratchetting strain is also overpredicted. Chaboche et al. [2-4] have proposed a decomposed nonlinear kinematic model, by superposing Armstrong-Frederick hardening rules. Three decomposed hardening rules proposed by Chaboche have been used in the present study to simulate ratchetting. The material constants associated with the Chaboche model can be derived easily from a uniaxial stable hysteresis loop [5].

Garud et al. [6], Hassan et al. [7], Lang et al. [8], Mahbali and Eslami [9], Xia and Elliyn [10] and Yoshida [11] have compared numerical results with experiments under cyclic loadings. Ohno and co-authors [12-14] have also reported various numerical studies under mechanical and thermal ratchetting.

The theory of the Chaboche nonlinear kinematic hardening model, available in the ANSYS software package [15], is discussed briefly in the present paper. Materials like SA-333 Carbon steel and SS-304 Stainless steel are typically used in Nuclear Power Plants in India. Thus, ratchetting simulation has been performed using the Chaboche model for these materials to understand their behavior under uniaxial and biaxial loading conditions. The data obtained from the following three sets of experiments have been used for comparison.

(a) Strain controlled and stress controlled uniaxial tests on tensile specimens made of SA-333 Gr.6 carbon steel;

(b) Three point and four point bend tests on straight pipes made of SA333 Gr.6 carbon steel, subjected to constant internal pressure and cyclic bending load; and

(c) A shake table test of a pipe elbow of SS-304 stainless steel.

The strain controlled stable hysteresis loop has been used to calculate the Chaboche parameters. The return mapping approach with consistent elasto-plastic tangent moduli has been used in ANSYS [15] for numerical integrations of the constitutive equation.

\section{Chaboche model}

The rate independent version of the nonlinear kinematic hardening model proposed by Chaboche [2-4] has been considered here, which primarily involves superposition of three Armstrong-Frederick kinematic hardening rules. The kinematic hardening rule contains a 'recall term', which incorporates the fading memory effects of the strain path. The constitutive equation is based on linear isotropic elasticity, a von-Mises yield criterion and the associated flow rule.

The evolution equation for the backstress, $\dot{\alpha}$, proposed by Armstrong-Frederick [1] is given by

$\dot{\alpha}=\frac{2}{3} C \dot{\varepsilon}^{p}+\gamma \alpha \dot{p}$

Three decomposed rules of the Chaboche model can be expressed by superposing the backstress term as

$\{\alpha\}=\sum_{i=1}^{3}\{\alpha\}_{i}$
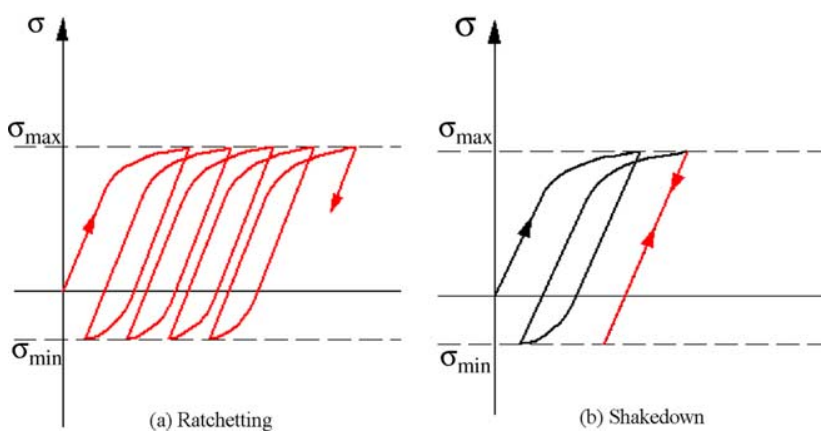

Fig. 1. Response of structure subjected to a repetitive cyclic load. 


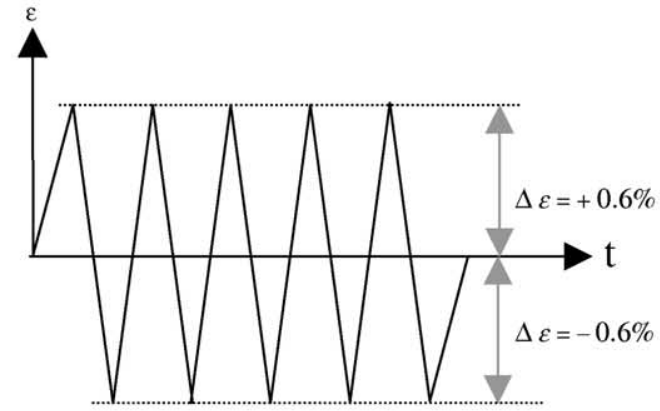

(a)

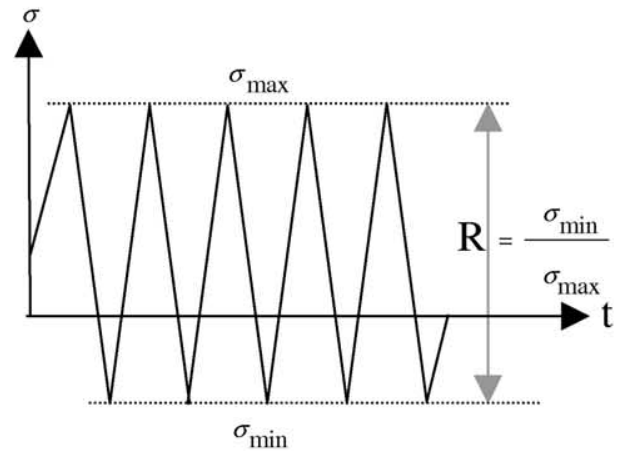

(b)

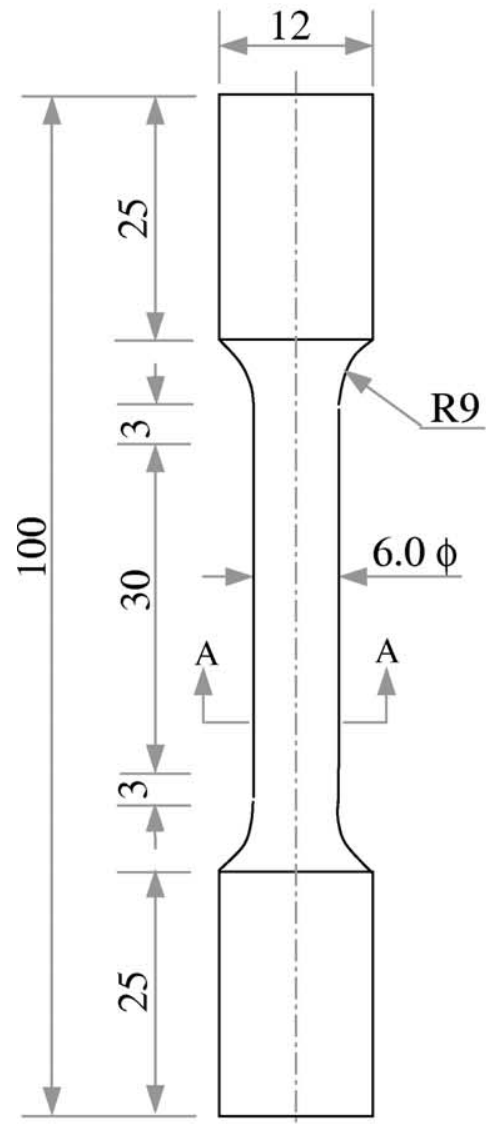

(c)

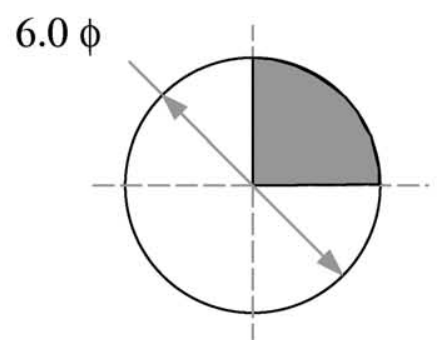

Section A-A

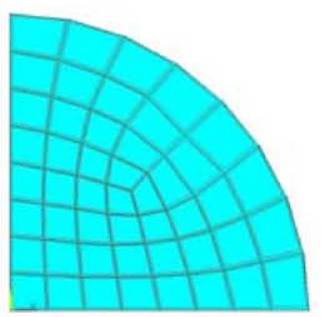

Section B-B

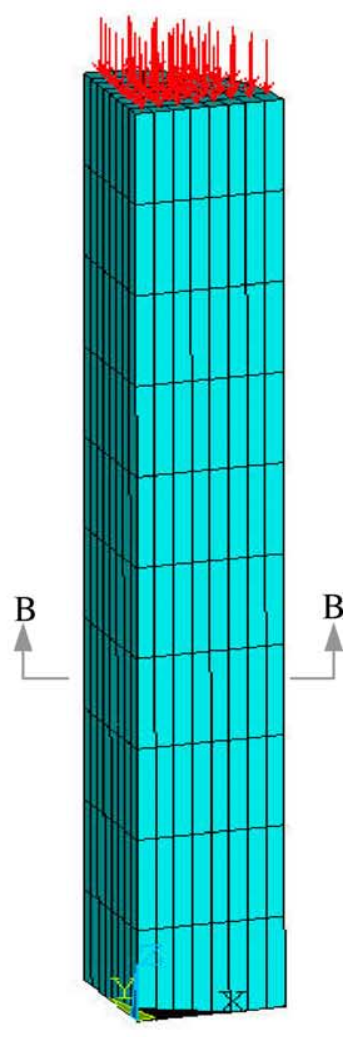

(d)

Fig. 2. Uniaxial test details (a) loading history during strain controlled test; (b) loading history during stress controlled test; (c) uniaxial specimen; and (d) finite element model of the specimen.

Each of the three decomposed rules described by Eqs. (1) and (2) thus divides the hysteresis loop into three segments and has its own specific purpose in simulating the ratchetting phenomenon. Chaboche suggested that the first rule $\left(\alpha_{1}\right)$ should comprise the initial steep part of the stable stress-strain curve reaching a very large modulus and stabilizing thereafter. The second rule $\left(\alpha_{2}\right)$ would simulate the transient hardening portion of the stable hysteresis loop and the third rule $\left(\alpha_{3}\right)$ would represent the linear part of the stable hysteresis loop at high strain values. The evolution of backstress (the kinematic hardening rule) for each component is defined by

$$
\{\dot{\alpha}\}_{i}=\frac{2}{3} C_{i}\left\{\dot{\varepsilon}^{p}\right\}+\gamma_{i}\{\alpha\}_{i} \dot{p}
$$


where $C_{i}$ and $\gamma_{i}$ are constants in the Chaboche kinematic hardening model, which can be evaluated from the uniaxial strain controlled stable hysteresis loop. The value of the parameter $\gamma_{3}$ is determined from an uniaxial ratchetting experiment ( $\varepsilon_{x}$ vs $N$ plot) to produce the best possible fit. For the value of $\gamma_{3}=0$ (linear third rule) ratchetting ceases completely (shakedown). A slight nonlinearity assigned to the third rule by a small value will improve ratchetting simulation. The detailed physical description and the calculation procedure of the Chaboche parameter is discussed in [5]. The parameter $\dot{p}$ is the plastic strain rate expressed as

$\dot{p}=\sqrt{\frac{2}{3} \dot{\varepsilon}^{p}: \dot{\varepsilon}^{p}}$

An additive decomposition of the strain rate tensor, $\dot{\varepsilon}$, is assumed such that

$\dot{\varepsilon}=\dot{\varepsilon}^{e}+\dot{\varepsilon}^{p}$

Here $\dot{\varepsilon}^{e}$ represents the elastic component of the strain rate tensor and $\dot{\varepsilon}^{p}$ represents the corresponding plastic strain rate component.

According to the normality condition, the associated flow rule can be described by the expression

$$
\left\{\dot{\varepsilon}^{p}\right\}=\lambda\left\{\frac{\partial f}{\partial \sigma}\right\}
$$

The von-Mises yield criterion is expressed as

$f(\sigma-\alpha)=\left[\frac{3}{2}(s-a):(s-a)\right]^{1 / 2}=k$

where, $\sigma$ is the stress tensor, $s$ is the deviatoric stress tensor, $\alpha$ is the current centre of the yield surface and a is the current centre of the yield surface in deviatoric space. The parameter $k$ in Eq. (7) is the radius of the yield surface. The initial size of the yield surface is given by $k_{o}$, which defines the initial yield strength of the material in an uniaxial tension test.

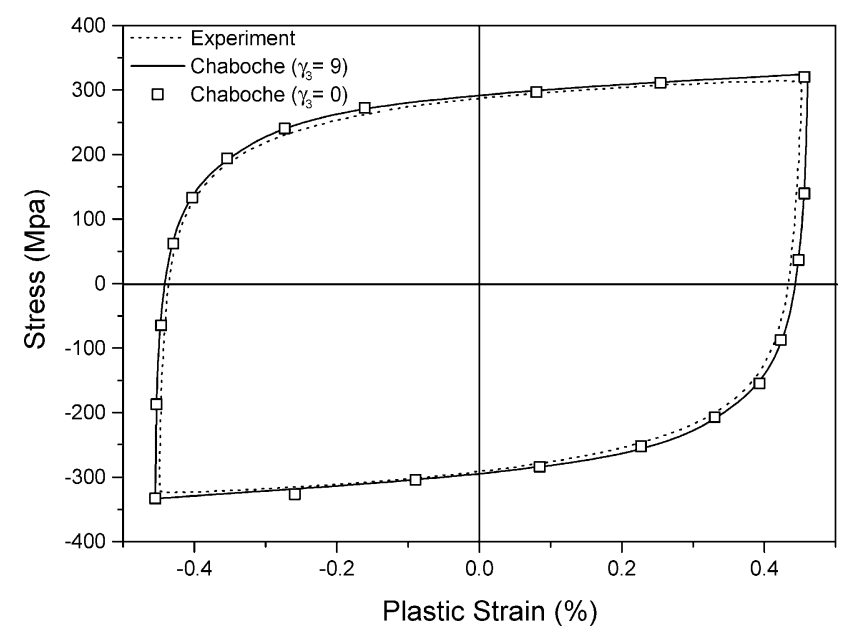

Fig. 3. Prediction from the Chaboche model with three decomposed rule for stable hysteresis loop under strain controlled test.
Table 1

Chaboche parameters

\begin{tabular}{llllllllll}
\hline Materials & \multicolumn{1}{l}{ Parameters } & & & & & & \\
\cline { 2 - 8 } & $C_{1}$ & $C_{2}$ & $C_{3}$ & $\gamma_{1}$ & $\gamma_{2}$ & $\gamma_{3}$ & $E$ & $\mu$ \\
\hline $\begin{array}{l}\text { SA-333 Gr. 6 } \\
\text { Carbon Steel }\end{array}$ & 112,3000 & 50,500 & 5900 & 280,750 & 950 & 9 & 203,400 & 0.3 \\
$\begin{array}{l}\text { SS-304 } \\
\text { Stainless Steel }\end{array}$ & 108,5000 & 43,000 & 4100 & 271,250 & 800 & 9 & 193,060 & 0.3 \\
\hline
\end{tabular}

In the above formulation, (:) indicates the second product between the second rank tensor and (.) indicates the derivate with respect to time.

\section{Experiments and numerical simulation}

Experimental and numerical simulations were performed for uniaxial specimens, straight pipes and an elbow as mentioned in Section 1. Uniaxial experiments have been performed at the material level, where the state of stress is uniform everywhere except near the ends. The cyclic

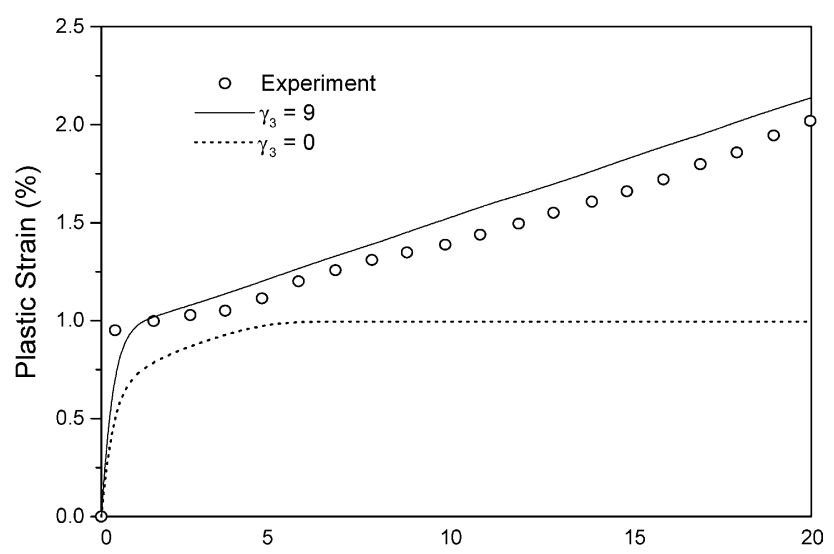

(a)

No. of Cycles

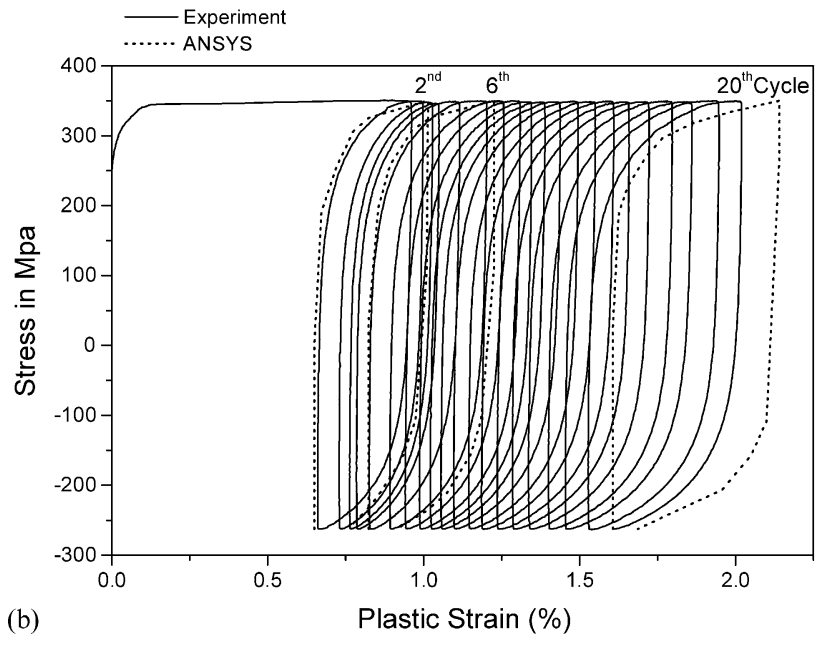

Fig. 4. Prediction from the Chaboche model with three decomposed rule for (a) stress-controlled hystersis loop; and (b) axial strain at positive stress peaks of uniaxial cycles. 
bending and the shake table tests have been conducted at the structural level where the state of stress varies from point to point. While selecting the test specimens for the uniaxial and the biaxial tests, care has been taken to ensure uniformity in the material properties. The details of the tests and their simulation with the analysis are discussed next.

\subsection{Uniaxial tests on tensile specimens}

Uniaxial cyclic experiments were performed under strain controlled (Fig. 2a) and stress controlled (Fig. 2b) conditions on $6 \mathrm{~mm}$ diameter tensile specimens made of SA333 Gr. 6 carbon steel (Fig. 2c). The test specimens were machined from long tubular stock with $450 \mathrm{~mm}$ outside diameter and $31 \mathrm{~mm}$ wall thickness. A $25 \mathrm{kN}$ capacity servo-hydraulic machine was used. A $25 \mathrm{~mm}$ gauge extensometer was attached to the tensile specimens to measure the strain during the tests. The strain controlled tests imparted the specimen with symmetric tensioncompression cycles within the strain limits $\Delta \varepsilon= \pm 0.6 \%$. The stabilized hysterisis plot of $\sigma-\varepsilon^{\mathrm{p}}$, obtained from the test is shown in Fig. 3. This plot was used to calculate the Chaboche parameter constants- $\left(C_{1}, \gamma_{1}\right),\left(C_{2}, \gamma_{2}\right)$ and $\left(C_{3}, \gamma_{3}\right)$ by trial and error in accordance with the procedure given by Shafiqul and Hassan [5]. The values of the parameters are listed in Table 1. These parameters have

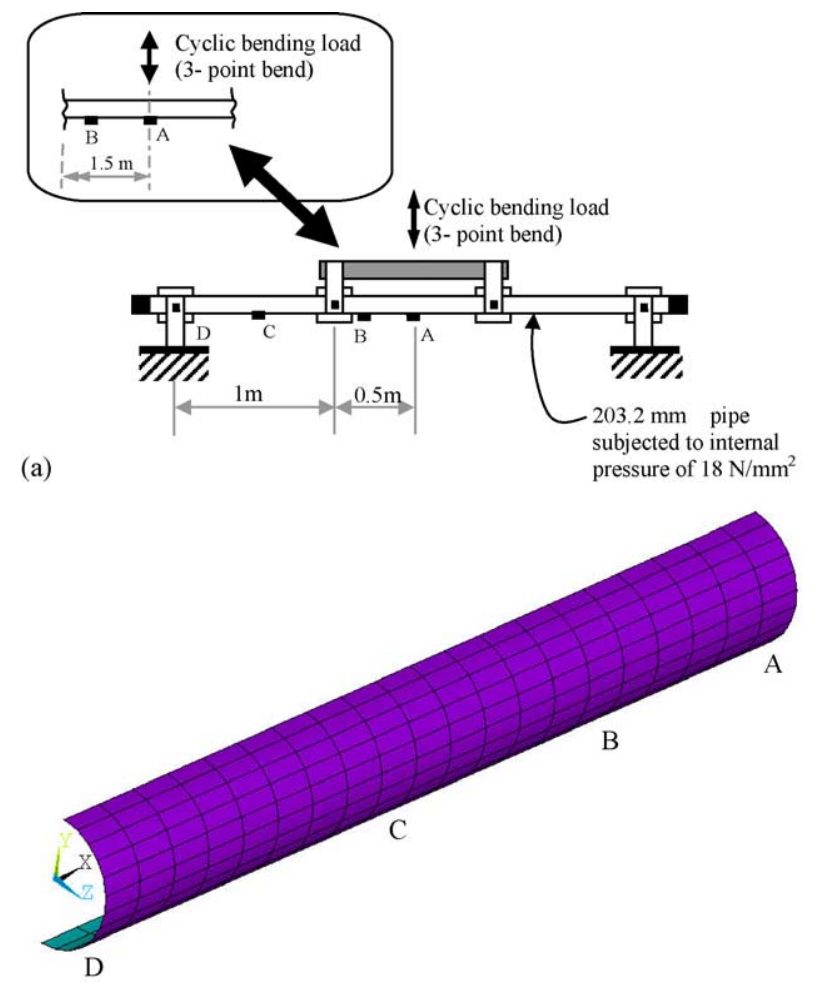

(b)

Fig. 5. Four-point and three-point bend test of straight pipe under constant internal pressure and cyclic load (a) schematic of the test setup; and (b) finite element model. been employed in numerical simulation of the cyclic behavior using the Chaboche model implemented in ANSYS [15]. The geometric model of a quarter of the tensile specimen using SOLID-68 elements was found to be sufficient for the FEM simulation due to the double symmetry. Results of the simulations with the experimental hysteresis plot have been compared in Fig. 3. It can be observed from the figure that numerical results closely follow the $\sigma-\varepsilon^{p}$ plot obtained from the constant strain tests.

The cyclic load applied to the specimen varied between two fixed nominal stress limits in the stress-controlled experiment. The upper stress limit, $\sigma_{\max }$, was kept at a constant value of $350 \mathrm{MPa}$ and the stress ratio, $R$, was maintained at -0.75 , where $R=\left(\sigma_{\min } / \sigma_{\max }\right)$ and $\sigma_{\min }$ is
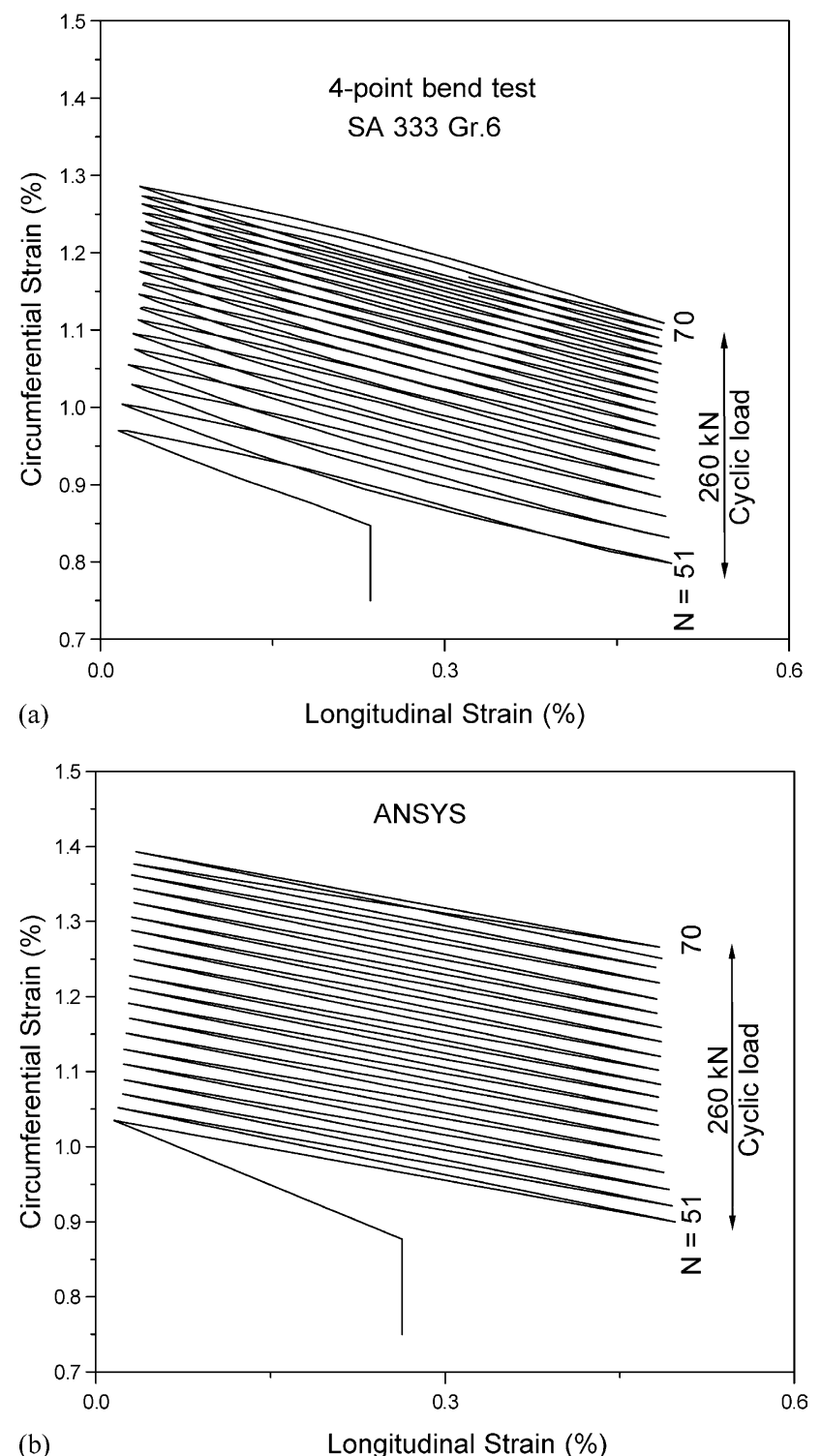

Fig. 6. Circumferential strain v/s axial strain for four-point bend test on straight pipe subjected to constant internal pressure of $18 \mathrm{MPa}$ and cyclic bending load (a) test results; and (b) results obtained from ANSYS. 


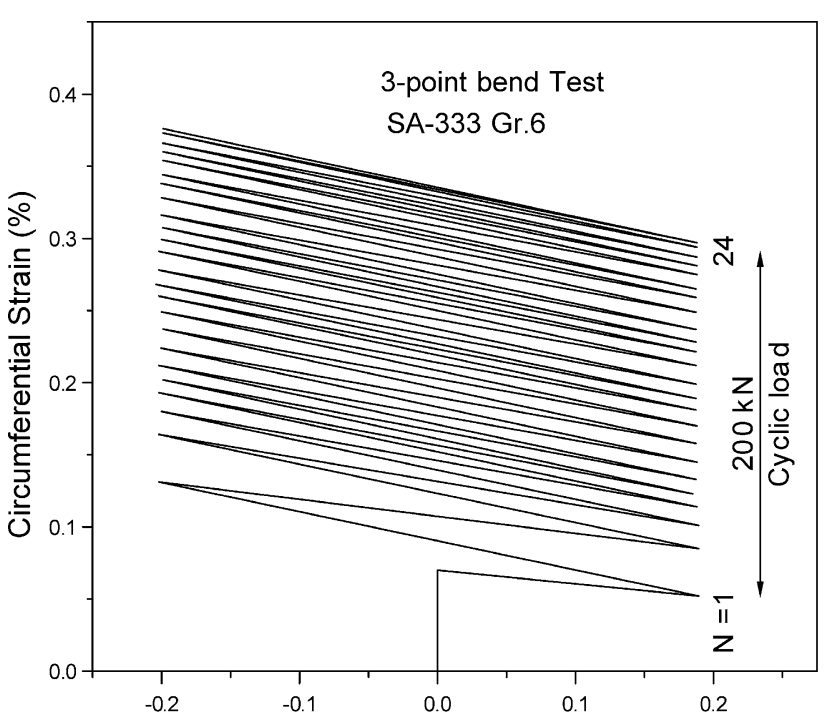

(a) Longitudinal strain (\%)

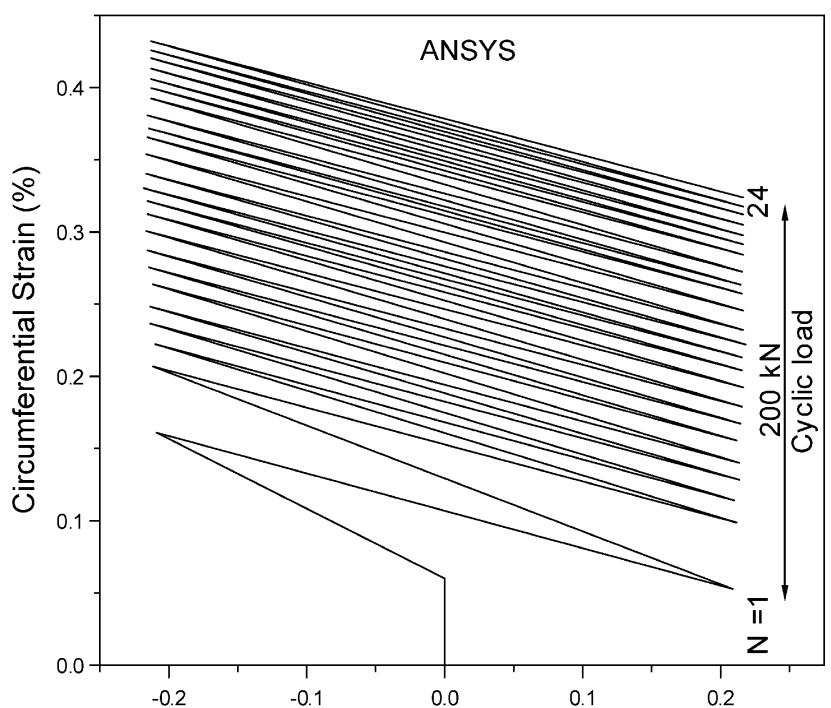

(b)

Longitudinal Strain (\%)

Fig. 7. Circumferential strain v/s axial strain for three-point bend test on straight pipe subjected to constant internal pressure of $18 \mathrm{MPa}$ and cyclic bending load (a) test results; and (b) results obtained from ANSYS.

the lower stress limit. Comparison of the ratchetting strain by the Chaboche model with the experimental result is shown in Fig. 4. The model predicted ratchetting for the first five cycles for $\gamma_{3}=0$. However, the ratchetting strain gradually reduces and the test approached complete shakedown (Fig. 4(a)), which is in contrast to the experimental trend. The calibration for both the materials has been performed using different values of $\gamma_{3}$ and it has been observed that $\gamma_{3}=9$ provide reasonably close simulation as presented in Fig. 4(a). However, the change in the value of $\gamma_{3}$ does not introduce any noticeable change in the stable hysteresis loop in a strain-controlled test (see Fig. 3). Thus the calibration of the parameter $\gamma_{3}$ is important for improving uniaxial ratchetting simulation. The stresscontrolled hystersis loop at the second, sixth and 20th cycles obtained from the experiment is compared in Fig. 4(b) with those given by the Chaboche model. The hysteresis loop obtained by the Chaboche model traces the experimental loop very well. However, it has been found that the Chaboche model somewhat overpredicts the ratchetting strain.

\subsection{Cyclic bending at constant internal pressure}

The set of tests involved both three-point and four-point stress controlled cyclic bending of straight pipes subjected to constant internal pressure. The specimens were $203.2 \mathrm{~mm}$ diameter, schedule 80, SA-333 Gr.6 carbon steel having $3.0 \mathrm{~m}$ length. A schematic of the test set-up for a four-point bend test is shown in Fig. 5. A cyclic load was applied at a distance of 1 and $2 \mathrm{~m}$ from the support. A similar test set-up was used for the three-point bend test with cyclic load applied at the centre of the pipe. Cyclic bending loads of $\pm 240 \mathrm{kN}$ (50 cycles) and $\pm 260 \mathrm{kN}$ (40 cycles) were applied in four-point bend tests, whereas a cyclic load of $\pm 200 \mathrm{kN}$ was applied in the three-point bend test. A constant internal pressure of $18 \mathrm{MPa}$ was maintained throughout both tests. More details about the test setup and the experimental
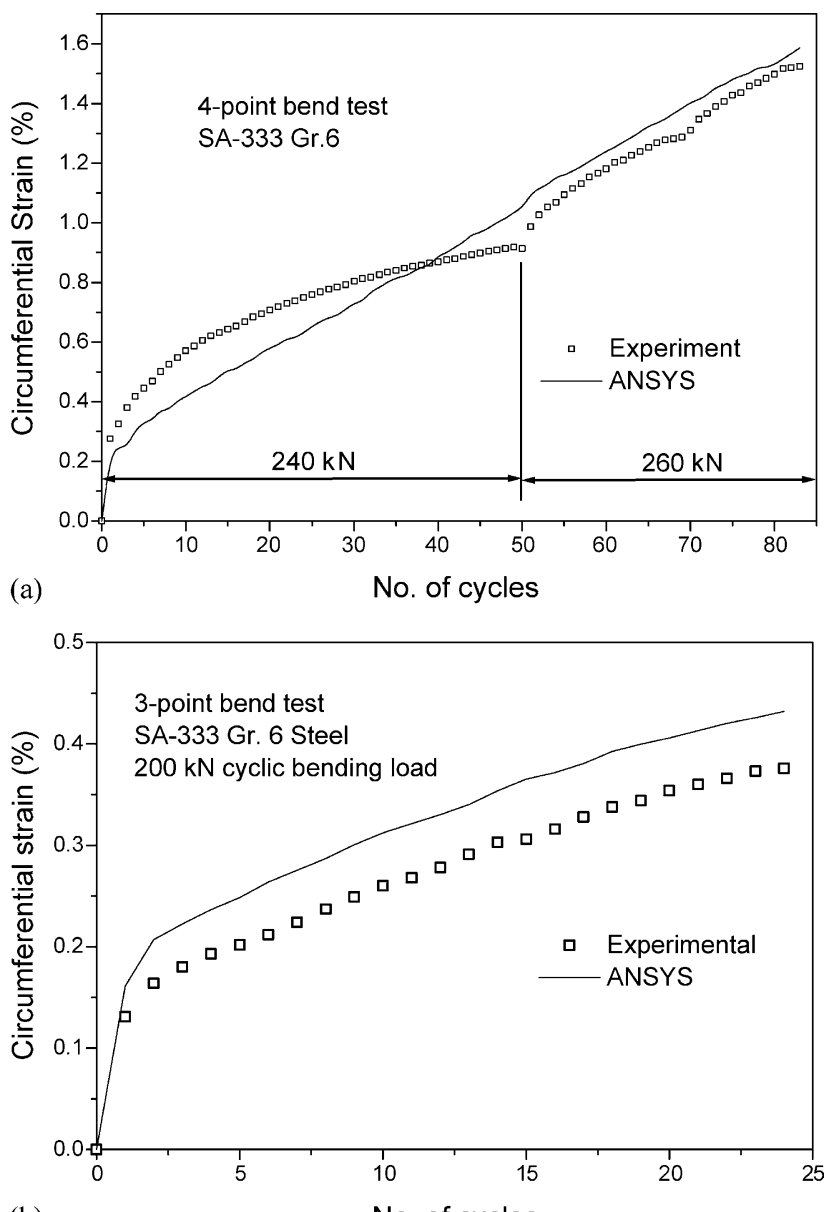

(b)

No. of cycles

Fig. 8. Circumferential ratchetting strain v/s number of cycles (a) four-point bend test; and (b) three-point bend test. 
(a)

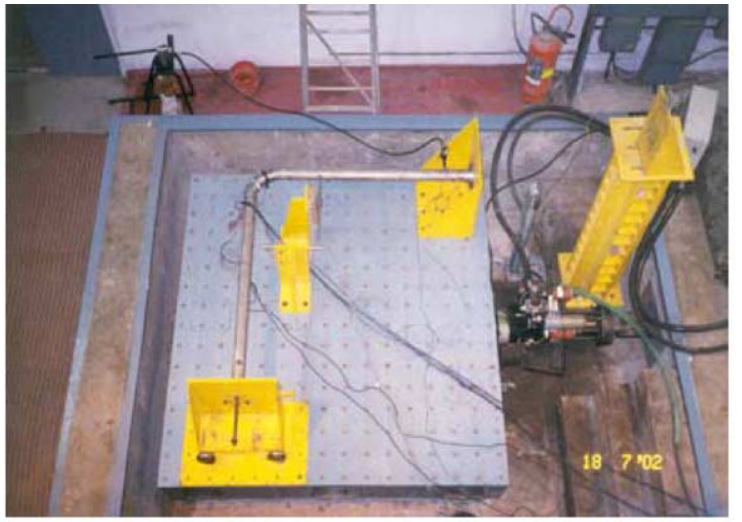

(c)

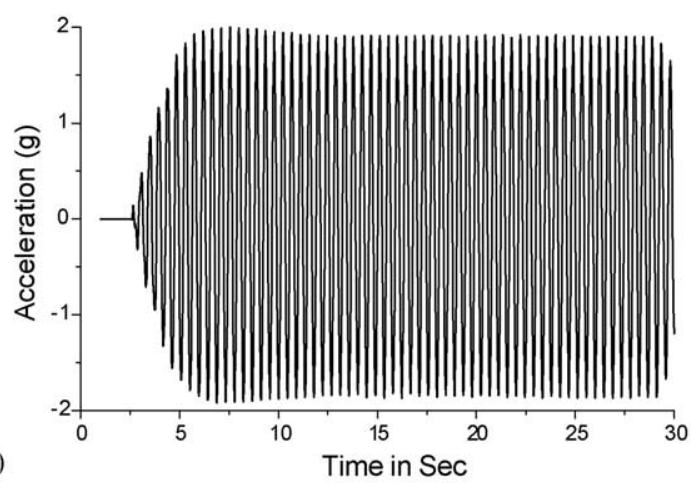

(b)
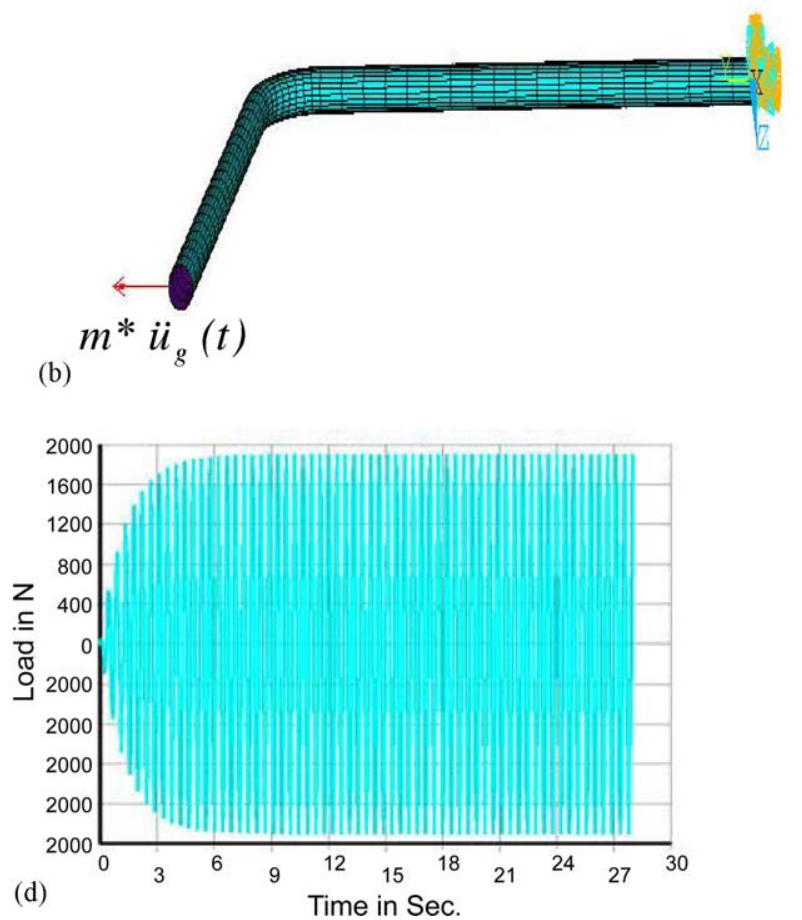

Fig. 9. Elbow pipe analysis (a) experimental test setup; (b) finite element model consider in ANSYS; (c) cyclic acceleration applied at the tip of pipe in experimental study; and (d) cyclic load consider in analysis.
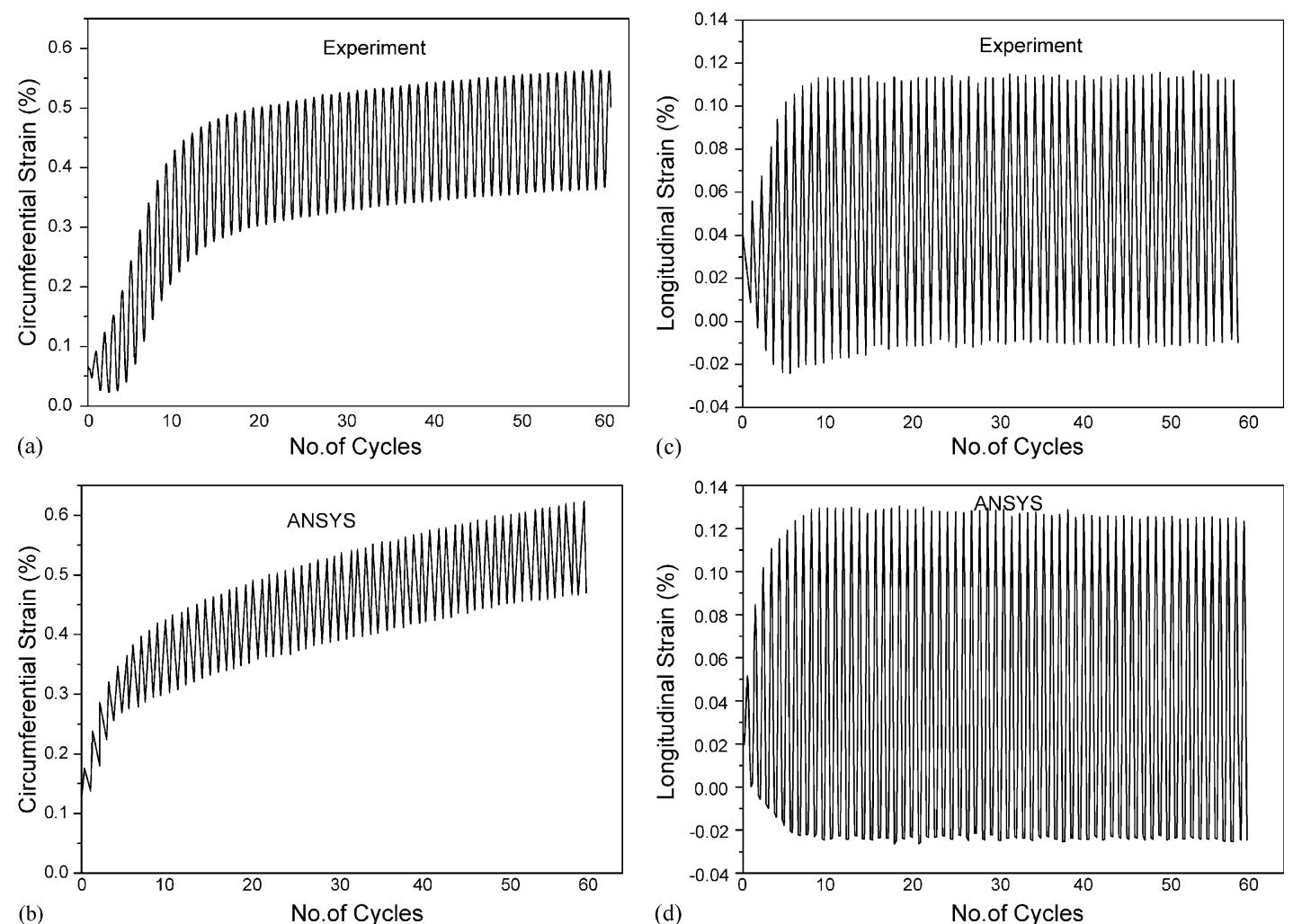

Fig. 10. Shake table analysis of elbow subjected to constant internal pressure of $18 \mathrm{MPa}$ and cyclic excitation (a) circumferential strain-experiment; (b) circumferential strain obtained from ANSYS; (c) longitudinal strain-experiment; and (d) longitudinal strain obtained from ANSYS. 
results are discussed in [16]. Typical test results of the axial and circumferential strain at location ' $A$ ' in the four-point and three-point bend tests are presented in Figs. 6(a) and 7 (a), respectively. The experimental results indicate that ratchetting occurs in the circumferential direction for a pipe subjected to constant internal pressure and cyclic bending load.

Modeling of the pipe has been done in ANSYS [15] utilizing the symmetry of the problem in both the longitudinal and circumferential directions. The finite element mesh comprising SHELL-181 elements is formed of $12 \times 20$ elements in the circumferential and longitudinal directions, respectively, and is shown in Fig. 5. The strains were calculated by using the Chaboche material parameters in Table 1.

The experimental results obtained in the form of circumferential v/s longitudinal strain for the four-point bend test under a cyclic bending load of $260 \mathrm{kN}$ are compared with the numerical results obtained using the Chaboche model in Fig. 6(b). A similar plot for the threepoint bend test with a $200 \mathrm{kN}$ cyclic bending load is shown in Fig. 7(b). Fig. 8(a) and (b) indicate circumferential strain accumulation with number of cycles for four-point and three-point bend test. Results presented in Fig. 8(a) for the four-point bend test indicate that the Chaboche model initially underpredicts ratchetting for the first 40 cycles. However, the simulation compares reasonably well with the experimental results at higher loading cycles. On the other hand, the Chaboche model overpredicts ratchetting by about $14 \%$ compared to the experimental results for the threepoint bend test, as indicated by Fig. 8(b).

\subsection{Set-up for shake table test on elbow pipe}

The test was performed on a shake table $(1.2 \mathrm{~m} \times 1.0 \mathrm{~m})$ coupled with a servo-controlled electro-hydraulic actuator of $100 \mathrm{kN}$ capacity. A large radius pipe elbow of diameter $76.2 \mathrm{~mm}$, schedule 80, SS-304, stainless steel was used. On either end of the elbow, two straight arms, each $1.5 \mathrm{~m}$ long were welded. One end of the pipe elbow was rigidly connected to the shake table, whereas the other end was kept free and attached with a mass of $100 \mathrm{~kg}$. Steady state cyclic excitation tests were conducted on the specimen. An internal pressure of $18 \mathrm{MPa}$ was maintained during the cyclic excitation. The details of the pipe set-up are shown in Fig. 9(a). The pipe elbow was modeled in ANSYS [15] by using SHELL-181 plastic elements as shown in Fig. 9(b). The cyclic acceleration applied during the experiment and corresponding cyclic force applied during the analysis is shown in Fig. 9(c) and (d), respectively. The material parameters $C_{i}$ and $\gamma_{i}$ for SS-304 steel were evaluated from the cyclic stress strain curve and are given in Table 1.

Fig. 10(a) and (c) indicate the circumferential and longitudinal strain accumulation with time for the first 60 cycles during shake-table test. The corresponding results predicted by the Chaboche model are shown in Fig. 10(b)

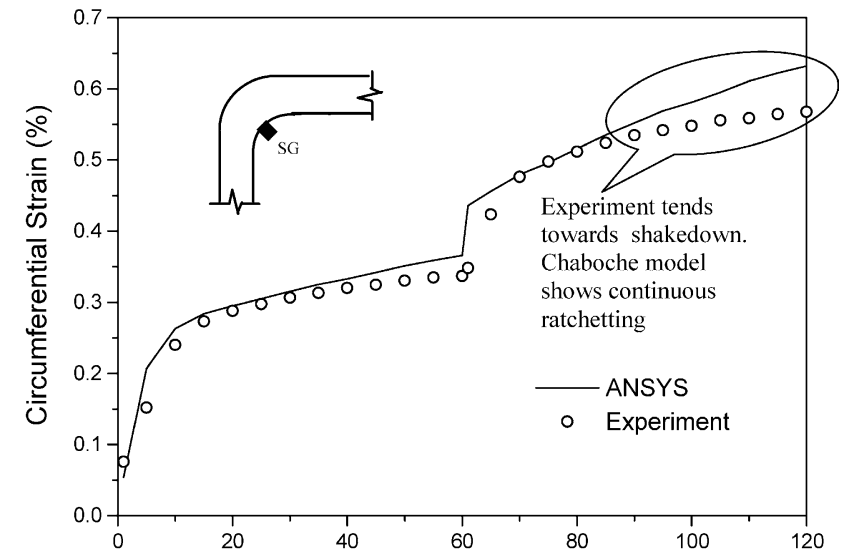

(a)

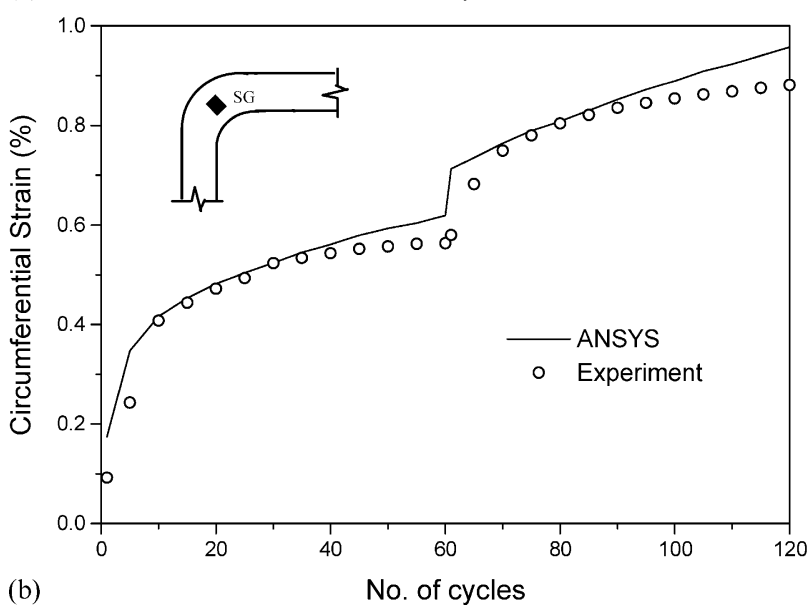

Fig. 11. Comparison of the circumferential strain accumulation of elbow pipe for the first 120 cycles: Experiment and analyses results (a) at the intrados of elbow; and (b) at $90^{\circ}$ from the intrados of elbow.

and (d). The numerical results obtained by the Chaboche model show ratchetting in the circumferential direction only, with no strain accumulation in the longitudinal direction and thus follow the experimental trend. The accumulation of the circumferential strain with number of cycles at the intrados and at the crown of the elbow are presented in Fig. 10.

The ratchetting strain obtained using the Chaboche model has been found to be very close to the experimental results. However, the experimental results indicate a decreasing rate of ratchetting beyond 100 cycles. The Chaboche model, on the other hand, indicates continuous ratchetting. In light of these observations, it can be stated that improvement in the decayed term of the Chaboche model is needed for simulating the condition of reduced rate of ratchetting with increasing number of cycles as observed in the experiments (Fig. 11).

\section{Conclusions}

Performance of the Chaboche model available in ANSYS in predicting ratchetting has been discussed in 
this paper. The results have been compared with three sets of test-data, viz. (a) uniaxial cyclic tests; (b) three-point and four-point bending tests; (c) a shake table test on a pipe elbow. The Chaboche kinematic hardening model has been used to predict ratchetting under cyclic loading with nonzero mean load. The parameters of the Chaboche model have been calculated from the stable hysteresis loop. The following conclusions can be drawn from various investigations performed in the present paper:

a. The Chaboche model with the linear third rule $\left(\gamma_{3}=0\right)$ has resulted in shakedown with persistent cycling. Incorporation of nonlinearity into the third rule by assigning a small value to $\gamma_{3} \quad\left(\gamma_{3}=9\right.$ in present investigation) improves the model's capability of simulating steady rate ratchetting and prevents shakedown. However this small value of $\gamma_{3}$ does not introduce any noticeable change in the strain controlled stable hysteresis loop simulation.

b. The Chaboche model has been found to predict ratchetting in the circumferential direction alone with no strain accumulation in the longitudinal direction for three-point and four-point bend analysis and a shake table analysis of an elbow, and predictions from the model follow the experimental trend.

c. It has been observed that the Chaboche model underpredicts ratchetting for the four-point bend analysis as compared to the experimental values in the initial cycles. However, good correlation with the experimental results is obtained at higher cycles. On the other hand, the Chaboche model overpredicts ratchetting compared to the experimental values in three-point bend analysis.

d. The Chaboche model has nicely correlated the experimental data for the shake table analysis except for long-term overprediction at higher number of cycles. Further improvement in the decaying term of the Chaboche model is needed for simulating the reduced rate of ratchetting at higher cycles.

\section{Acknowledgements}

The work presented here has been sponsored by the Board of Research in Nuclear Sciences, India (BRNS Grant No. 99KA012). The authors acknowledge with thanks help rendered by Dr Satish Kumar (IIT Madras), Mr M.A. Khan,
Mr S.N. Bodele and Mr K.K. Bavu, Bhabha Atomic Research Center (BARC) in conduct of the biaxial pipe test. The authors are grateful to Mr N Gopalkrishanan and other staff at Structural Engineering Research Centre, Chennai for their help in the conduct of the shake table experiment. Also, invaluable discussions and suggestions by Shri. H.S. Kushwaha (BARC) and Dr M.S. Sivakumar (IIT Madras) are gratefully acknowledged. Constructive comments of reviewer is also acknowledged.

\section{References}

[1] Armstrong PJ, Frederick CO. A mathematical representation of the multiaxial baushinger effects. CEGB Report 1966, RD/b/N/731, Berkeley Nuclear Laboratories, Berkeley, UK.

[2] Chaboche JL. Time independent constitutive theories for cyclic plasticity. Int J Plasticity 1986;2:384-92.

[3] Chaboche JL, Nouailhas D. Constitutive modeling of ratchetting effects, Part I and II. ASME J Engng Mater Technol 1989;111: 384-92. see also pages 409-416.

[4] Chaboche JL. On some modification of kinematic hardening to improve the discribtion of ratchetting effects. Int J Plasticity 1991;7: $661-78$.

[5] Bari S, Hassan T. Anatomy to coupled constitutive models for ratchetting simulation. Int J Plasticity 2000;16:381-409.

[6] Garud YS, Durlofsky H, Tagart SM. Analysis and prediction of fatigue ratchetting; comparison with tests and code rules. J Strain Anal 1996;31:135-51.

[7] Hassan T, Zhu Y, Matzen V. Improved ratchetting analysis of piping components. Int J Press Vessels Piping 1998;75:643-52.

[8] Lang H, Wirtz K, Heitzer M, Staat M, Oettel R. Cyclic plastic deformation tests to verify FEM-based shakedown analyses. Nucl Engng Design 2001;206:235-47.

[9] Mahbali H, Eslami MR. Cyclic loading of beams based on the Prager and Frederick-Armstrong kinematic hardening models. Int J Mech Sci 2004;

[10] Xia Z, Ellyin F. Biaxial ratchetting under strain or stress-controlled axial cycling with constant hoop stress. ASME J Appl Mech 1994;61: 422-8.

[11] Yoshida F. A constitutive model of cyclic plasticity. Int J Plasticity 2000;16:359-80.

[12] Ohno N. Constitutive modeling of cyclic plasticity with emphasis on ratchetting. Int J Mech Sci 1998;2-3:251-61.

[13] Kobayashi M, Ohno N. Thermal ratchetting of a cylinder subjected to a moving temperature front; effects of kinematic hardening rules on the analysis. Int J Plasticity 1996;12:255-71.

[14] Mizuno M, Mima Y, Abdel-Karim M, Ohno N. Uniaxial ratchetting of 316FR steel at room temperature: part I and II. J Engng Mater Technol 2000;122:29-34. see also pp. 35-41.

[15] ANSYS 2000. ANSYS user's Manual, Release 6. Houston, USA: ANSYS Inc. Providence.

[16] Kulkarni SC, Desai YM, Kant T, Reddy GR, Parulekar Y, Vaze KK. Uniaxial and biaxial ratchetting studies of SA 333 Gr. 6 at room temperature. Int J Press Vessels Piping 2003;80:179-85. 